\title{
A Higher-Order Ray Approximation Applied to Orographic Gravity Waves: Gaussian Beam Approximation
}

\author{
MANUEL PULIDO \\ Department of Physics, FACENA, Universidad Nacional del Nordeste, Corrientes, and CONICET, Buenos Aires, Argentina \\ Claudio Rodas \\ Department of Physics, FACENA, Universidad Nacional del Nordeste, Corrientes, Argentina
}

(Manuscript received 10 February 2010, in final form 21 May 2010)

\begin{abstract}
Ray techniques are a promising tool for developing orographic gravity wave drag schemes. However, the modeling of the propagation of orographic waves using standard ray theory in realistic background wind conditions usually encounters several regions, called caustics, where the first-order ray approximation breaks down. In this work the authors develop a higher-order approximation than standard ray theory, named the Gaussian beam approximation, for orographic gravity waves in a background wind that depends on height. The analytical results show that this formulation is free of the singularities that arise in ray theory. Orographic gravity waves that propagate in a background wind that turns with height - the same conditions as in the work of Shutts-are examined under the Gaussian beam approximation. The evolution of the amplitude is well defined in this approximation even at caustics and at the forcing level. When comparing results from the Gaussian beam approximation with high-resolution numerical simulations that compute the exact solution, there is good agreement of the amplitude and phase fields. Realistic orography is represented by means of a superposition of multiple Gaussians in wavenumber space that fit the spectrum of the orography. The technique appears to give a good representation of the disturbances generated by flow over realistic orography.
\end{abstract}

\section{Introduction}

Momentum flux divergences produced by unresolved gravity waves are modeled in climate models by means of parameterizations. The effects of these small-scale waves appear to be so ubiquitous and systematic that particular patterns in the zonal mean general circulation cannot be modeled properly in climate and weather forecast models without considering them. For instance, aspects of the temperature and surface pressure fields (Palmer et al. 1986; McFarlane 1987) and the closing of the mesospheric jets (Lindzen 1981) are not well represented. Furthermore, recent studies suggest that unresolved gravity wave drag, which is prescribed by parameterizations, plays an important role in driving the strength of the BrewerDobson circulation (Alexander and Rosenlof 1996;

Corresponding author address: Manuel Pulido, Facultad de Ciencias Exactas, UNNE, Av. Libertad 5400, Corrientes 3400, Argentina.

E-mail: pulido@exa.unne.edu.ar
Pulido and Thuburn 2008) and its response to climate change (Li et al. 2008; McLandress and Shepherd 2009). Therefore, the correct representation of gravity wave effects is essential to obtain conclusive results.

In general there are two kinds of gravity wave parameterizations in climate models. The first represents the effects of waves generated by subgrid orography (e.g., Lott and Miller 1997; Webster et al. 2003). These parameterizations are assumed to represent waves with zero absolute frequency. The second kind (known as nonorographic gravity wave parameterizations) is assumed to represent all the rest of the frequency spectrum (e.g., Hines 1997; Scinocca 2003). In general both kinds of parameterizations assume steady-state and only vertical propagation.

There are several studies that model the propagation and dissipation of the wave field by means of ray theory (e.g., Marks and Eckermann 1995; Bühler et al. 1999; Broutman et al. 2006; Hasha et al. 2008). In this approach both horizontal and vertical wave propagation are modeled, including the refraction of the wave field by horizontal background wind changes, so that many of 
the assumptions of current gravity wave parameterizations may be relaxed under ray theory. Wave propagation from localized and transient sources can be modeled properly under this representation. This approach appears to be one of the most promising for future parameterizations to represent realistically gravity wave effects in climate models.

A wave train is a system of waves that has a dominant wavenumber. The amplitude may vary with the position but only slowly compared to the dominant wavelength (Bretherton and Garrett 1968). We refer to this as the slowly varying wave train approximation. Ray theory is based on the slowly varying wave train approximation in which the approximated solution is expressed in the form

$$
\Phi(x, y, z)=A(x, y, z) \exp \left[i \epsilon^{-1} \psi(x, y, z)\right]
$$

where $\Phi$ is a wave field (e.g., vertical displacement), $A$ is an amplitude function that needs to be determined, $\phi \equiv$ $\epsilon^{-1} \psi$ is the phase, and $\epsilon$ is a small nondimensional parameter. The phase may depend nonlinearly on $x, y$, and $z$. Bretherton (1969) introduces the wave train analysis for internal gravity waves and discusses in detail the formal expansion in the small parameter $\epsilon$.

In this work we deal with gravity waves generated by steady mean flows over fixed orography. The solution is then time independent, hence the approximated solution (1). In time-dependent problems the functions $A$ and $\phi$ in (1) are also a function of $t$.

The wave equations may be expressed as an asymptotic series in the small parameter $\epsilon$. In the limit $\epsilon \rightarrow 0$ the resulting first-order equations for $A$ and $\psi$ are dominant and are known in general as the transport and eikonal equations, respectively (e.g., Lewis 1965). The solutions for these first-order equations give $A$ and $\psi$, which in (1) represent a good approximate solution of the wave problem. The transport and eikonal equations for the gravity wave equations are obtained in Tanushev et al. (2007).

For nonuniform background flows, ray theory needs a further assumption that the spatial scale of background flow changes must be longer than the dominant wavelength of the wave train. This assumption is known as the Wentzel-Kramers-Brillouin-Jeffries (WKBJ) approximation. In terms of gravity wave parameterizations this scale separation hypothesis of ray theory is in accord with the gravity wave parameterization assumptions. The parameterizations assume that the well-resolved spectrum of climate models may be considered as the mean flow upon which the unresolved small-scale waves are propagating. These small-scale waves are the ones modeled by the parameterization.
First-order ray theory when representing the wave field in the whole physical domain is only valid far from the source or from the forcing boundary. To overcome this limitation, Broutman et al. (2001) suggested integrating the full linear solution near the source, which is obtained under the WKBJ approximation. The stationary phase approximation of this near-field solution is used to initialize the ray tracing technique that is then used far from the sources. The ray tracing technique implemented in Broutman et al. (2001) includes the calculation of wave action density.

However, there is another problem that appears in spatial ray theory far from the source. Rays focus in regions where the volume of ray tubes becomes zero; in these regions the amplitude contains a singularity under spatial ray theory. Such regions are usually called "caustics" (e.g., Lighthill 1978). Caustics have a simple physical interpretation in the context of electromagnetic theory: they are the boundary of shadow regions. In the neighborhood of caustics spatial ray solutions break down and singularities appear. The singularities are an artifact of the approximation and are absent at higher-order approximations.

Some of the state-of-the-art ray techniques (e.g., Marks and Eckermann 1995; Hasha et al. 2008) make a strong approximation: the cross-sectional area of ray tubes is constant along the entire ray. The cross-sectional area is taken perpendicular to the rays that compose the thin tube. The assumption of constant area avoids the appearance of caustics.

There are some attempts to represent more precisely the wave amplitude evolution using the area of ray tubes given by ray theory where the area is nonzero. At the points where the cross-sectional area of ray tubes is zero a caustic exists. Therefore, an alternative method needs to be proposed to calculate the wave field at caustics and their neighborhood. Broutman et al. (2002) propose using Maslov's method to calculate the wave field at the singularity points. In this method the wave field is represented locally in the neighborhood of caustics as the Maslov integral. The Maslov integral represents the wave field in the neighborhood of caustics as the Fourier transform from wavenumber space to physical space of the ray theory approximated solution.

This work proposes an alternative methodology to solve the breakdown of spatial ray theory in both situations, near sources and near caustics. The suggestion is to use a higher-order approximation in the asymptotic expansion instead of using the full linear integral solution or the Maslov integral. In this sense, the phase function is represented as in ray theory and stationary phase method up to the second order in a Taylor series around the characteristic/central wavenumber. On the other hand, the amplitude function is represented as an exact solution 
to the slowly varying wave train problem. A simpler application of this methodology is used in Pulido and Rodas (2008) for transient gravity waves propagating in a shear flow. A particular comparison with the full linear integral solution shows that the proposed approximation-the Gaussian beam approximation-gives encouraging results. In particular, the short time behavior and the behavior near the critical levels are well modeled.

In standard ray theory, the wave field is determined by the contribution of wave packets that are assumed to be concentrated in space and propagate along rays. The wave packets are assumed to be composed locally of a single monochromatic wavenumber, the central wavenumber. On the other hand, the Gaussian beam approximation assumes that the wave field is composed locally of a Gaussian spectrum centered at the central wavenumber. This Gaussian spectrum generates a beam of rays around the central one; in this way diffractive effects are considered in this approximation. In this sense, the Gaussian beam approximation explicitly deals with the width of the wave packet and therefore the conservation of the wave action in a ray beam is considered in a self-consistent way.

The Gaussian beam approximation is used extensively in other contexts, such as electromagnetism (e.g., Heyman and Felsen 2001) and quantum mechanics [the treatment of Gaussian states-so-called coherent states-for the time-dependent Schrödinger equation is developed in Hagedorn (1985) and references therein]. There are also mathematically rigorous results by Hagedorn (1984) on the convergence of the approximation for the nondispersive wave equation in inhomogeneous media.

Gaussian beam solutions for mountain waves are also examined in Tanushev et al. (2007). A formal proof is derived there, showing that the system is well posed and that the Gaussian beam stationary solution is close to the time-dependent full integral solution.

\section{Mathematical formulation}

Consider orographic waves forced by mountains whose horizontal scales are such that rotation and nonhydrostatic effects may be neglected. The forced waves propagate in a time-independent horizontally uniform mean flow that depends on height, $\mathbf{U}=[U(z), V(z)]$. An approximated solution may be found if the mean flow changes slowly with height compared to the vertical wavelength. This is the well-known WKBJ approximation that, in the examined case, is satisfied for large Richardson number, $\mathrm{Ri}=N^{2} /\left|d_{z} \mathbf{U}\right|^{2} \gg 1$, where the Brunt-Väisälä frequency $N$ is constant. Under this approximation the local vertical wavenumber of stationary gravity waves forced by mean flow over orography is given by

$$
m=\frac{N|\mathbf{k}|}{\mathbf{U} \cdot \mathbf{k}}
$$

The horizontal wavenumber is $\mathbf{k}=(k, l)$. The sign of $m$ in (2) is the same as the sign of $\mathbf{U} \cdot \mathbf{k}$ so that only waves propagating upward are considered. The relation (2) obtained under the WKBJ approximation excludes wave reflections.

The vertical displacement for a single sinusoidal wave in the WKBJ approximation is

$\eta(x, y, z)=\eta_{0}\left(\frac{m}{m_{0}}\right)^{1 / 2} \exp \left\{i\left[k x+l y+\int_{0}^{z} m\left(z^{\prime}\right) d z^{\prime}\right]\right\}$

where $m_{0}=m(z=0)$ is the vertical wavenumber at the bottom boundary. This particular WKBJ solution (3) has been extensively used in previous works (e.g., Shutts 1998; Broutman et al. 2002). Hereinafter we deal with complex expressions; physical quantities must be interpreted as the real part of the complex expressions.

The general solution for forced waves generated by an isolated mountain or a mountain range may be determined by a linear superposition of (3),

$$
\begin{aligned}
\eta(x, y, z)= & \iint \hat{\eta}(k, l) \Theta\left(m / m_{0}\right)\left(\frac{m}{m_{0}}\right)^{1 / 2} \\
& \times \exp \left\{i\left[k x+l y+\int_{0}^{z} m\left(z^{\prime}\right) d z\right]\right\} d k d l
\end{aligned}
$$

where $\hat{\eta}(k, l)$ is determined by the bottom boundary condition and $\Theta$ is the Heaviside function. This Heaviside function eliminates the sinusoidal waves that encounter a critical level above that level. Since the radiation condition has been used, only waves propagating upward are represented in (4).

We consider a small-amplitude mountain height $h(x, y)$ so that we impose the linearized bottom boundary condition on (4) at $z=0$. The resulting bottom boundary condition in wavenumber space is

$$
\hat{\eta}(k, l)=\hat{h}(k, l)
$$

where $\hat{h}(k, l)$ is the two-dimensional Fourier transform of $h(x, y)$.

Equation (4) represents the general solution to the linear conservative system under the WKBJ approximation. However, the integral (4) can only be solved analytically in highly simplified cases. From the numerical point of view, the calculation of (4) is a very expensive method to be implemented as a parameterization of subgrid scales in a climate model. The alternative is to make assumptions about the spectral amplitude $\hat{\eta}(k, l)$ in order to solve the 
integral analytically. There is a well-known method of approximating this integral: ray theory. Strictly, ray theory and the related equation that governs wave amplitude, known as the wave action conservation equation (Bretherton and Garrett 1968), are derived using the slowly varying wave train assumption in physical space (see, e.g., Bretherton 1969).

The slowly varying wave train assumption taken in ray theory imposes a restriction on the amplitude of the disturbance. The envelope amplitude of the disturbance must vary slowly compared to the wavelength scale so that the width of the envelope encompasses several wavelengths. This assumption can also be interpreted in wavenumber space; that is, the spectrum of the disturbance must be locally a narrow peak around the characteristic wavenumber. The mapping between points in wavenumber space and points in physical space is, therefore, justified. As will be seen below, this assumption is also a central hypothesis in the Gaussian beam approximation.

Under the slowly varying wave train assumption, the disturbance may be represented locally as a plane wave with a well-defined wavenumber. If the phase of the disturbance is $\phi$, the local wavenumber is given by

$$
\mathbf{K}=-\nabla \phi
$$

The propagation of the disturbance is concentrated in space along well-defined paths:

$$
d_{z} X=\left(d_{z} x, d_{z} y\right)=\left(-\partial_{k} m,-\partial_{l} m\right) .
$$

The derivatives $\partial_{k} m, \partial_{l} m$ in (7) along the spatial rays represent the local dispersion rates in physical space of a group of waves. These derivatives may be considered as the "group velocity" for a time-independent problem. Note that, since we examine stationary gravity waves generated by a time-independent mean flow over orography, the concept of wave group velocity does not need to be introduced. Ray tubes extend from the sources to the sinks independently of time.

Although ray theory and the stationary phase method in particular are well known in fluid dynamics (e.g., Lighthill 1978), we give here a short description of the methods with a unified notation so that the similarities and differences with the Gaussian beam approximation are more clearly established.

\section{a. Method of stationary phase}

We take $s$ as the length of the ray, that is, $d s=\left(d x^{2}+\right.$ $\left.d y^{2}+d z^{2}\right)^{1 / 2}$, as the independent parameter, which is expected to be arbitrarily large. This parameter plays the same role as $t$ in the standard stationary phase method for transient wave disturbances (Lighthill 1978). The solution (4) is expressed as

$$
\eta(x, y, z)=\iint \tilde{\eta}(k, l, z) \exp [i \phi(k, l, z)] d k d l,
$$

where $\phi=k x+l y+\int_{0}^{z} m\left(z^{\prime}\right) d z^{\prime}$ is the phase. We are interested in the asymptotic behavior of the integral (8) when $s \rightarrow \infty$ for fixed values of $x / s, y / s$ and $\int_{0}^{z} m\left(z^{\prime}\right) d z / s$.

Under the WKBJ approximation, the spectral amplitude in (8), from (4), is

$$
\tilde{\eta}(k, l, z)=\hat{\eta}(k, l) \Theta\left(m / m_{0}\right)\left(\frac{m}{m_{0}}\right)^{1 / 2} .
$$

The functions $\tilde{\eta}(k, l, z)$ and $\phi(k, l, z)$ are expanded as a Taylor series around the central wavenumber $\mathbf{k}_{c}$ of the wave train found at $(x, y, z)$. We only take the first term in the Taylor series of the amplitude:

$$
\tilde{\eta}(k, l, z) \approx \tilde{\eta}_{c} .
$$

Hereinafter, the subscript $c$ means evaluation at the central wavenumber; for example, $\tilde{\eta}_{c} \equiv \tilde{\eta}\left(k_{c}, l_{c}\right)$.

Since the exponential is a rapidly varying function, the method takes up to second order in the Taylor series of the phase

$$
\phi(k, l, z) \approx \phi_{c}+\frac{1}{2} \partial_{k_{i} k_{j}}^{2} \phi_{c}\left(k_{i}-k_{c i}\right)\left(k_{j}-k_{c j}\right)+\cdots,
$$

where the usual suffix convention is used-that is, a suffix that appears twice within a term must be interpreted as a sum over the index range, $\sum_{i=1}^{2} a_{i} b_{i} \equiv a_{j} b_{j}$.

The integration of (8) for large $s$ is dominated by the stationary phase points, $\partial_{k} \phi_{c}=0$. In general, sinusoidal waves that compose the integral (8) cancel by destructive interference, except at stationary phase points. Then, the asymptotic value for large $s$ of the integral in (8) is entirely determined by the stationary phase points. A formal proof of this result may be derived using Cauchy's theorem (e.g., Lighthill 1978). The Taylor series of the phase (11) is taken around $\mathbf{k}_{c}$ along the curve where the stationary phase points are located, $\partial_{k_{i}} \phi_{c}=0$.

Then, replacing (10) and (11) in (8), the asymptotic wave field yields

$$
\begin{aligned}
\eta(x, y, z)= & \tilde{\eta}_{c} \exp \left(i \phi_{c}\right) \\
& \times \int_{0}^{\infty} \int_{0}^{\infty} \exp \left[i \frac{1}{2} \partial_{k_{i} k_{j}}^{2} \phi_{c}\left(k_{i}-k_{c i}\right)\left(k_{j}-k_{c j}\right)\right] d k_{1} d k_{2} .
\end{aligned}
$$

Solving the Gaussian error integral in (12) gives 
$\eta(x, y, z)=2 \pi\left(\partial_{k k}^{2} \phi_{c} \partial_{l l}^{2} \phi_{c}-\partial_{k l}^{2} \phi_{c}\right)^{-1 / 2} \tilde{\eta}_{c} \exp \left(i \phi_{c}\right)$

where $\tilde{\eta}_{c}=\hat{\eta}_{c}\left(m_{c} / m_{0 c}\right)^{1 / 2} \Theta\left(m_{c} / m_{0 c}\right)$.

\section{b. Amplitude using ray tubes}

Ray theory is based on the assumption that one can expand the amplitude term and the phase function as a power series of a small parameter. The exponential with the phase function as argument is assumed to be highly varying compared to the amplitude function. The energy of the disturbance components is then concentrated along the rays with a well-defined wavenumber. In this conservative system the horizontally integrated vertical wave action flux is constant, so for a thin ray tube the vertical wave action flux times the area of the ray tube horizontal cross section is constant (Lighthill 1978).

A simple proof of the wave amplitude dependence under the assumptions of ray theory is found using the Parseval theorem in a neighborhood of the point in consideration. The main contribution to the amplitude in wavenumber space is coming from the spectral amplitude of the mode whose ray crosses the point in consideration. The relation between the square of the amplitude in physical space and the power spectrum is found by multiplying (8) by $\eta(x, y, z)^{*}$ and integrating in space; this results in the Parseval theorem: ${ }^{1}$

$$
\iint|\eta|^{2} d x d y=(2 \pi)^{1 / 2} \iint|\tilde{\eta}|^{2} d k d l .
$$

The integral in the rhs of (14) is assumed to be dominated by the contributions around the central wavenumber $\mathbf{k}_{c}$, $\iint\left|\tilde{\eta}_{c}\right|^{2} d k d l \approx\left|\tilde{\eta}_{c}\right|^{2} \Delta k \Delta l$; then it is transformed from the wavenumber space $k, l$ to the physical space $x, y$

$$
|\eta(x, y, z)|^{2}=(2 \pi)^{2}\left|\tilde{\eta}_{c}\right|^{2}\left[\left.J\left(\frac{x, y}{k, l}\right)\right|_{c}\right]^{-1},
$$

where $J$ is the Jacobian of the transformation from the physical space to the wavenumber space evaluated at the central wavenumber. From (9), we obtain

$$
|\eta(x, y, z)|^{2}=(2 \pi)^{2}\left|\hat{\eta}_{c}\right|^{2} \frac{m_{c}}{m_{0 c}} \Theta\left(\frac{m_{c}}{m_{0 c}}\right)\left[J\left(\frac{x, y}{k, l}\right) \mid\right]_{c}^{-1} .
$$

\footnotetext{
${ }^{1}$ The expression of the Parseval theorem, (14), is in complete accordance with the conservation of the wave action. See Pulido (2005) for a discussion of the relation between the Parseval theorem and the wave action conservation equation. For the case in consideration, the wave action conservation may be expressed in wavenumber space as $[\mathbf{U}(z) \cdot \mathbf{k}]|\tilde{\eta}(k, l, z)|^{2}=$ const, where $\tilde{\eta}(k, l, z)$ is the 2D Fourier transform at $z$.
}

An alternative proof of (16) is given in Shutts (1998), using that the vertical component of the wave action flux is constant along a ray.

The Jacobian may be expressed as a function of the phase $\phi$. Using (7), the Jacobian yields

$$
J\left(\frac{x, y}{k, l}\right)=\partial_{k k}^{2} \phi \partial_{l l}^{2} \phi-\left(\partial_{k l}^{2} \phi\right)^{2} .
$$

Note that multiplying the stationary phase solution, (13), by its complex conjugate gives the same expression for the amplitude as in the ray tube approximation, (16).

\section{c. Gaussian beam approximation}

To derive the approximate solution under the Gaussian beam approximation we again consider a solution of the form (8) and assume that we are in an asymptotic regime where the $s$ parameter is large enough that the solution may be represented as the first terms in the asymptotic expansion. The Gaussian beam approximation is introduced by Cerveny et al. (1982) and Popov (1982) as a representation of seismic wave fields. Tanushev et al. (2007) give a formal proof of Gaussian beams as an approximate solution to the mountain wave field.

The amplitude in the wavenumber space is represented locally as a concentrated envelope, namely a Gaussian, around the central wavenumber $\mathbf{k}_{c}$; this is the wavenumber of the ray that is found at the position $(x, y, z)$ :

$$
\begin{aligned}
\tilde{\eta}(k, l)= & \hat{\eta}_{c}\left(\frac{m_{c}}{m_{0 c}}\right)^{1 / 2} \Theta\left(m_{c} / m_{0 c}\right) \\
& \times \exp \left[-\frac{\left(k-k_{c}\right)^{2}}{2 \sigma_{k}^{2}}-\frac{\left(l-l_{c}\right)^{2}}{2 \sigma_{l}^{2}}\right] .
\end{aligned}
$$

As in the stationary phase method, we approximate the phase function $\phi$ in (8) up to the second order in a Taylor series around the central wavenumber $\mathbf{k}_{c}$ :

$$
\begin{aligned}
\phi\left(k_{1}, k_{2}\right) \approx & \phi_{c}+\partial_{k_{i}} \phi_{c}\left(k_{i}-k_{c i}\right) \\
& +\frac{1}{2} \partial_{k_{i} k_{j}}^{2} \phi_{c}\left(k_{i}-k_{c i}\right)\left(k_{j}-k_{c j}\right)+\cdots .
\end{aligned}
$$

Contrary to the stationary phase method, in Gaussian beam approximation the first-order term in the Taylor series of the phase, (19), is nonzero. This is because the Gaussian beam approximation considers not only the contribution of the central ray but also a neighborhood of rays around it (the full beam of waves that compose the Gaussian).

The resulting integral, replacing (18) and (19) in (8), is 


$$
\eta(x, y, z)=\hat{\eta}_{c}\left(\frac{m_{c}}{m_{0 c}}\right)^{1 / 2} \Theta\left(m_{c} / m_{0 c}\right) \iint \exp \left[-\frac{1}{2}\left(\mathbf{k}-\mathbf{k}_{c}\right)^{\mathrm{T}} \mathbf{A}\left(\mathbf{k}-\mathbf{k}_{c}\right)+\mathbf{p}^{\mathrm{T}} \cdot\left(\mathbf{k}-\mathbf{k}_{c}\right)\right] d k d l
$$

where matrix $\mathbf{A}$ and vector $\mathbf{p}$ are defined as

$$
\mathbf{A}=\left[\begin{array}{cc}
\sigma_{k}^{-2}-i \partial_{k k} \phi_{c} & -i \partial_{k l} \phi_{c} \\
-i \partial_{l k} \phi_{c} & \sigma_{l}^{-2}-i \partial_{l l} \phi_{c}
\end{array}\right] ; \quad \mathbf{p}=\left[\begin{array}{c}
i \partial_{k} \phi_{c} \\
i \partial_{l} \phi_{c}
\end{array}\right]
$$

The integral in (20) can be solved exactly by completing squares and diagonalizing matrix $\mathbf{A}$ (e.g., Greiner and Reinhardt 1996); the result is

$$
\begin{aligned}
\eta(x, y, z)= & 2 \pi \hat{\eta}_{c}\left(\frac{m_{c}}{m_{0 c}}\right)^{1 / 2} \frac{\Theta\left(m_{c} / m_{0 c}\right)}{\sqrt{\operatorname{det} \mathbf{A}}} \\
& \times \exp \left[i \phi_{c}+\frac{1}{2} \mathbf{p}^{\mathrm{T}} \mathbf{A}^{-1} \mathbf{p}\right]
\end{aligned}
$$

where

$$
\operatorname{det} \mathbf{A}=\sigma_{k}^{-2} \sigma_{l}^{-2}-i b-J, \quad b=\frac{\partial_{k k} \phi_{c}}{\sigma_{l}^{2}}+\frac{\partial_{l l} \phi_{c}}{\sigma_{k}^{2}},
$$

and $J=\partial_{k k} \phi_{c} \partial_{u} \phi_{c}-\left(\partial_{k l} \phi_{c}\right)^{2}$ is the Jacobian (17).

The solution is composed of an amplitude term and an oscillatory term (exponential with the imaginary term). The last is the carrier wave. In turn, the amplitude is formed by the vertical wavenumber dependence, the inverse of the determinant of $\mathbf{A}$ and the Gaussian envelope.

This is the representation of the solution under Gaussian beam approximation. The fundamental difference with first-order ray theory is that the amplitude modulation of this representation is an exact solution of the Schrödinger equation (Hagedorn 1984; Ostrovsky and Potapov 1999). On the other hand, the ray tube approximation and stationary phase method are only the first-order solutions of the asymptotic expansion.

There is a more subtle difference between ray tube and Gaussian beam approximations, which is of paramount importance. The Gaussian beam approximation takes into account not only the central ray but also a beam of rays around the central one that are contributing to the wave field. In this way diffractive effects are included in the approximation. This beam of rays is essential to eliminate the singularities that arise in standard ray theory.

The stationary phase solution is by construction the dominant term far from the source for $s \rightarrow \infty$; in accordance with this, the Gaussian beam solution goes to the stationary phase solution in that limit. In other terms, the Gaussian beam solution is different from the stationary phase solution only where the stationary phase assumption is not satisfied; therefore, higher-order terms produce an important contribution. In the regions where the stationary phase solution is a good approximation, the Gaussian beam solution will be close to the stationary phase solution and only small differences coming from the small higher-order terms are expected. The stationary phase solution is recovered assuming that we have a wide Gaussian, $\sigma_{k}, \sigma_{l}, \rightarrow \infty$ in (18), so that the spectrum goes to a constant. In this limit (21) tends to (13). In general, for large $s$ and if the rays are separated, (13) is close to (21) (the $J$ term is dominant). On the other hand, when the rays converge to a caustic or diverge from the forcing level, $J \rightarrow 0$, then the other terms in (21) contribute significantly.

\section{Numerical simulations}

Several numerical simulations were performed in order to evaluate the Gaussian beam approximation in the context of orographic gravity waves generated by localized mountains. To compare the approximation with the exact solution, we use as the "exact solution" the numerical model described in Pulido (2005) that solves the Taylor-Goldstein equation, using a fourth-order RungeKutta algorithm, in the wavenumber space. The spectrum is resolved by $1024 \times 1024$ components. This high resolution is essential for the cases in which a critical level is present. The result is then Fourier transformed to the physical space. In what follows this high-resolution numerical solution is called the "exact case" (even though some numerical effects due to the application of the discrete Fourier transform may be found).

The numerical computation of the Gaussian beam approximation only involves an evaluation of (21). Note that (21) is expressed directly in physical space.

Two background conditions are considered: a constant wind and a wind turning with height (Shutts 1998). The last case has already been treated in several publications (e.g., Broad 1999; Broutman et al. 2001). It gives the possibility of testing the approximation under two characteristic features, caustics (Broutman et al. 2002) and multiple critical levels for which each wave that composes the spectrum has a critical level at a different height (these critical levels were called 3D critical levels by Broad).

A first set of experiments uses a Gaussian modulated wave of carrier wavenumber $\mathbf{k}_{c}$ (e.g., Martin and Lott 2007) as orographic forcing, which enables a direct comparison with the basic representation of the Gaussian beam approximation. Then non-Gaussian forcings, in particular a bell shape and a case study of realistic 
orography, are investigated through a superposition of Gaussians of the form (21). In the current algorithm each Gaussian contributes around its central ray until its amplitude contribution is negligible. Since the purpose of this study is the evaluation of the approximation in a few case studies, there is no need to further optimize the algorithm. In practical implementations, it may be worthwhile to consider the contribution of each Gaussian in a narrower spatial range around the central ray of the Gaussian, for example, $\sim 2 \sigma$.

\section{a. Single Gaussian beam}

\section{1) CONSTANT Wind}

The orographic forcing that produces the disturbance is a Gaussian modulated wave of carrier wavenumber $\mathbf{k}_{c}$, in the physical space. This Gaussian modulated sinusoidal wave is represented by

$$
h(x, y)=h_{0} \exp \left(-\frac{x^{2}}{2 \sigma_{x}^{2}}-\frac{y^{2}}{2 \sigma_{y}^{2}}\right) e^{i\left(k_{c} x+l_{c} y\right)} .
$$

This Gaussian modulated sinusoidal wave is the basic orographic forcing used in the Gaussian beam approximation. The Gaussian forcing generates a disturbance that is concentrated around the central ray.

For the orographic forcing (22), the spectrum is given by

$$
\tilde{\eta}_{0}(k, l)=\hat{\eta}_{c} \exp \left[-\frac{\left(k-k_{c}\right)^{2}}{2 \sigma_{k}^{2}}-\frac{\left(l-l_{c}\right)^{2}}{2 \sigma_{l}^{2}}\right]
$$

with amplitude $\hat{\eta}_{c}=h_{0} /\left(2 \pi \sigma_{k} \sigma_{l}\right)$. The central wavenumber is $k_{c}=2 \pi / 15 \mathrm{~km}, l_{c}=1.5 k_{c}$, and the widths $\sigma_{k}=\sigma_{x}^{-1}=2 \pi / 10 \mathrm{~km}$ and $\sigma_{l}=\sigma_{y}^{-1}=2 \pi / 10 \mathrm{~km}$. The disturbance propagates in a constant wind $U=10 \mathrm{~m} \mathrm{~s}^{-1}$, and the Brunt-Väisälä frequency is also set to a characteristic value, $N=0.01 \mathrm{~s}^{-1}$.

At the forcing level the Gaussian beam approximation (21) is exactly the Fourier transform of a Gaussian wavenumber spectrum, so there are no differences between the exact and the approximated solutions (Fig. 1). In other words, the full integral solution at $z=0$ is just the inverse Fourier transform of (23), which is exactly represented in (21).

As the disturbance propagates upward, the horizontal group velocity is southeast, as seen in Fig. 2, and the maximum amplitude of the disturbance is found at particular points of a parabola forming a localized branch of the characteristic $\mathrm{V}$-shape downstream pattern of isolated mountains. Higher-order dispersive effects take place at the head of the disturbance (the oscillations that are found farthest from the mountain at a given height). Because the

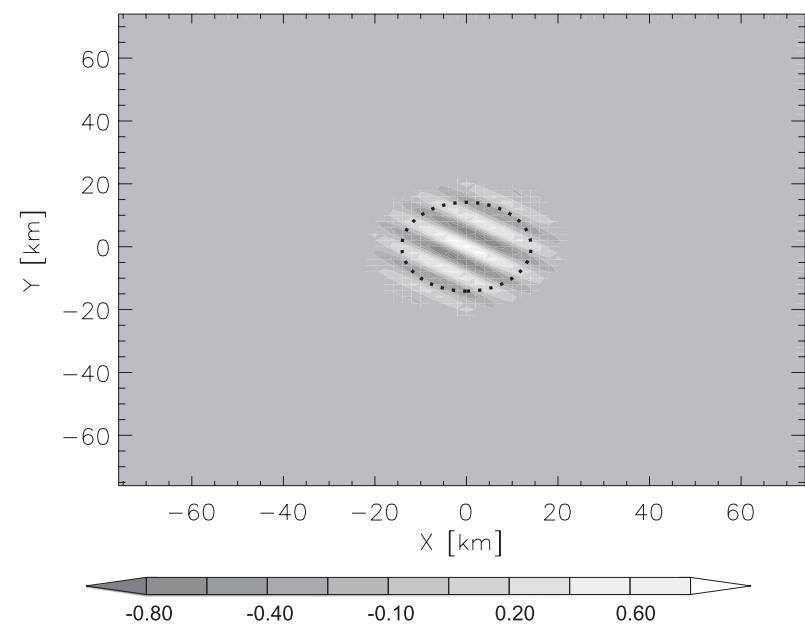

FIG. 1. Vertical displacement $\left(\eta / \eta_{c}\right)$ of the wave disturbance at the forcing level generated by a Gaussian modulated sinusoidal wave. The mountain is represented with the $\eta / \eta_{c}=\exp (-1)$ contour (dashed line).

Gaussian beam approximation only represents the dispersive effects up to second order, some slight differences in the exact solution may be detected there. On the other hand, around the location of the central ray the Gaussian beam approximation has a very precise representation of the disturbance. The lower panels of Fig. 2 show a $y-z$ cross section at $x=0$. The Gaussian beam approximation also presents a very good representation of the disturbance in both limits close and far from the source; only slight differences can be seen in Fig. 2.

\section{2) Wind TURNING WiTH HeIGHT: CAUSTICS}

We performed an experiment to evaluate the behavior of the Gaussian beam approximation in the presence of caustics. The background horizontal wind is constant in $x$ and linear in $z$, that is,

$$
\mathbf{U}=U_{0}\left[1,(2 \pi / \mathrm{km})^{-1} z\right]
$$

in which $U_{0}=10 \mathrm{~m} \mathrm{~s}^{-1}$, so that the wind vector is turning with height (left panel of Fig. 3). The wave forcing is a Gaussian spectrum whose the central wavenumber is given by

$$
\mathbf{k}_{c}=2 \pi /(20 \mathrm{~km})[\cos (0.108 \pi), \sin (0.108 \pi)]
$$

and $\sigma_{k}=\sigma_{l}=2 \pi /(10 \mathrm{~km})$. Rays belonging to this spectrum launched at $(x, y, z)=0$ find a caustic at $5 \mathrm{~km}$; the right panel of Fig. 3 shows a tube of rays that converge at the caustics. Note that to enhance the caustic effect a broad spectrum compared to its wavenumber 

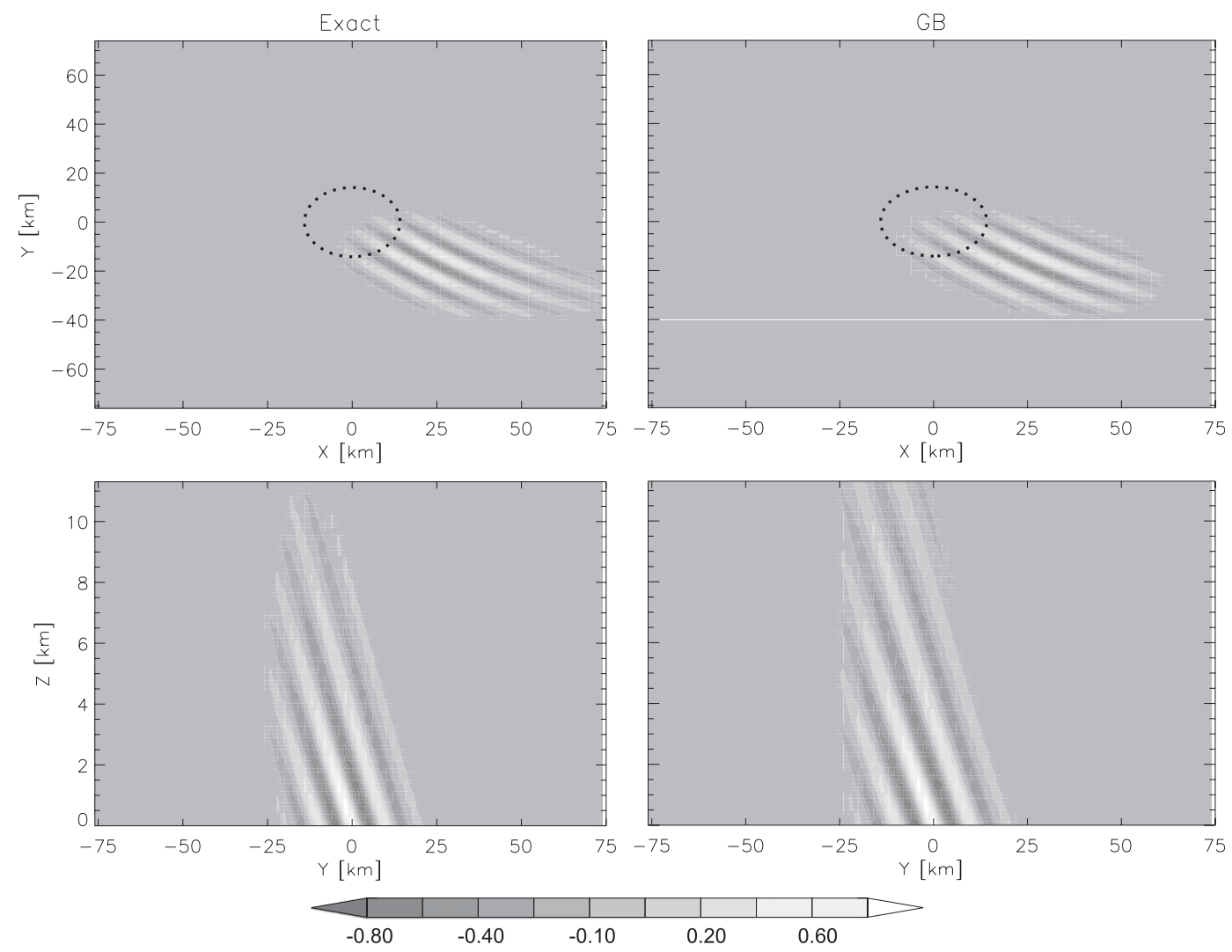

FIG. 2. Vertical displacement $\left(\eta / \eta_{c}\right)$ of the wave disturbance generated by Gaussian modulated sinusoidal wave orography: (top) horizontal cross section at $z=2.8 \pi U / N \approx 8.8 \mathrm{~km}$ and (bottom) a $y-z$ cross section at $x=0 \mathrm{~km}$ for the (left) exact solution and (right) approximation.

has been chosen. The existence of a caustic in this system is first mentioned and treated in Broutman et al. (2002).

At the caustics the Jacobian, (17), goes to zero; however, the term det $\mathbf{A}$, which is in the denominator of the Gaussian beam solution (21), is not zero, so the solution is well defined. Indeed, a cross section at the height of the caustics (Fig. 4) shows that the disturbance is a Gaussian wave packet with a slight deformation of the patterns coming from dispersion and diffraction. The Gaussian beam approximation (right panel of Fig. 4) gives almost exactly the wave disturbance pattern (left panel of Fig. 4) at the height of the caustic.

A vertical cross section is shown in Fig. 5. There are small refractive effects; the focusing of the rays in physical space is not found in the real disturbance. The Gaussian beam approximation (right panel of Fig. 5) represents very well the exact disturbance (left panel of Fig. 5) for this case where a caustic exists, and there are almost no visible differences between them (except for slight dispersive effects at high altitudes $-\sim 9 \mathrm{~km}$, far from the central ray-which are not well captured by Gaussian beam approximation).

\section{3) Wind turning With Height: Multiple CRITICAL LEVELS}

One of the simplest cases where multiple critical levels arise is the case of a wind in which the absolute value of the wind velocity is constant and the direction is turning with height (Broad 1999); that is,

$$
\mathbf{U}=U_{0}\left[\cos \left(\beta z+\phi_{0}\right), \sin \left(\beta z+\phi_{0}\right)\right],
$$

where $U_{0}=10 \mathrm{~m} \mathrm{~s}^{-1}, \beta=\pi / 10 \mathrm{~km}$, and $\phi_{0}=-\pi / 4$.

The wave forcing is a Gaussian spectrum (23) with $k_{c}=$ $2 \pi / 30 \mathrm{~km}, l_{c}=0$, and $\sigma_{k}=\sigma_{l}=1.3 k_{c}$. The sinusoidal wave with the central wavenumber encounters a critical level at $z_{l}=7.5 \mathrm{~km}$.

Figure 6 shows the exact case (left panel) and the Gaussian beam approximation (right panel). There are important differences in the neighborhood of the critical levels. The disturbance in the exact case is spread along the full height range of critical levels. This is because waves with higher critical levels than the central critical level give an important contribution to the disturbance 

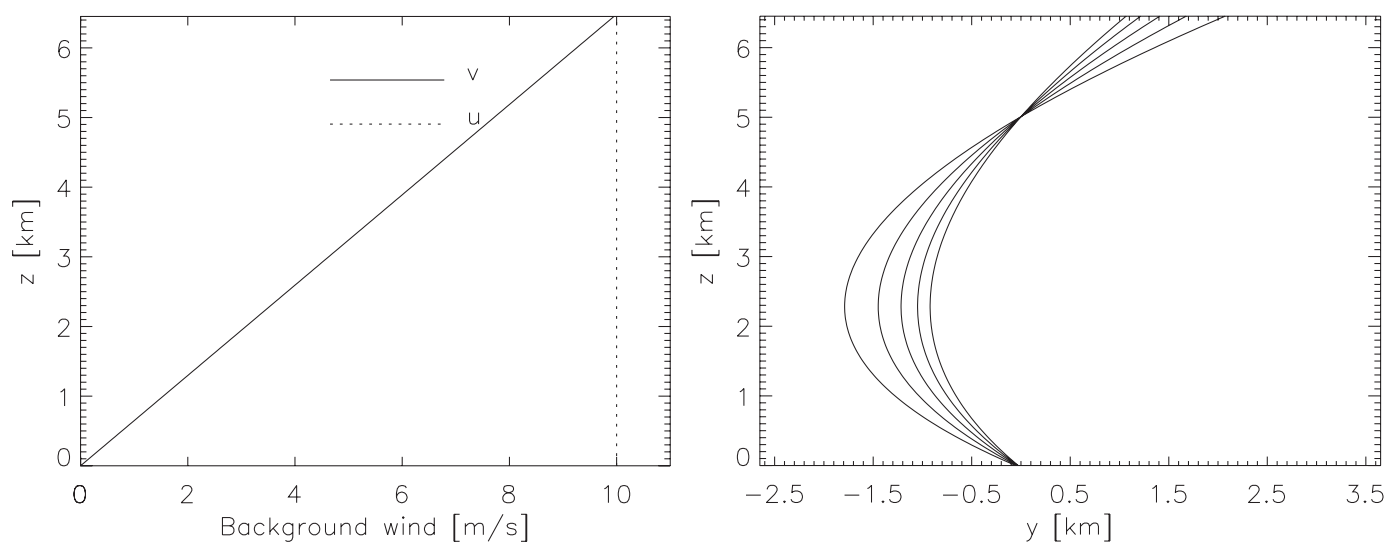

FIG. 3. (left) Background wind zonal (dashed line) and meridional (continuous line) components. (right) Rays of waves that belong to the disturbance in the $y-z$ plane at $x=0 \mathrm{~km}$.

amplitude near their own critical levels. The Gaussian beam approximation only accounts for the critical level of the central wavenumber; thus, the disturbance for large $s$ is concentrated below that critical level. No waves are found above that level. The Gaussian beam approximation is still a valid asymptotic expansion along the central ray and particularly near the central critical level, far from the source (say for $s \rightarrow \infty$ ), so that it gives a precise representation of the amplitude at the central ray.

\section{b. Superposition of Gaussians}

To examine more realistic cases a superposition of Gaussian beams is used. In general we use about 256 Gaussian beams to represent the disturbance at the forcing level. Once the characteristics of the Gaussian beams $\left(\hat{\eta}_{c}, \mathbf{k}_{c}, \sigma_{k}, \sigma_{l}\right)$ are determined, the wave disturbance is then calculated as a superposition of solutions of the form (21).
The procedure to construct the superposition of Gaussians in the wavenumber space is as follows. First, the wavenumber space is divided into grid cells of side $\Delta k$, $\Delta l$ in the region where nonnegligible spectral amplitudes are found. For instance, if we want a resolution of 256 Gaussians, the wavenumber region $\left(k_{\min }<k<k_{\max }\right.$ and $\left.l_{\min }<l<l_{\max }\right)$ is divided in 16 bins in the $k$ direction and 16 bins in the $l$ direction so that 256 cells are formed. The wavenumber at the center of each cell is $\left(k_{c i}, l_{c j}\right)=$ $\left[k_{\min }+\Delta k(i+1 / 2), l_{\min }+\Delta l(j+1 / 2)\right]$; then for each cell a Gaussian of the form (23) is constructed with central wavenumber $\left(k_{c i}, l_{c j}\right)$ and width $\left(\sigma_{k}, \sigma_{l}\right)=C(\Delta k, \Delta l)$, where $C$ is a constant. The Gaussian grid details and Gaussian locations are illustrated in Fig. 7.

To calculate the amplitude $\hat{\eta}_{c i j}$ of the Gaussian, the orography $h(x, y)$ is Fourier transformed to obtain $\hat{h}(k, l)$. For idealized cases we calculate the analytical spectrum; in realistic cases we use the fast Fourier transform to

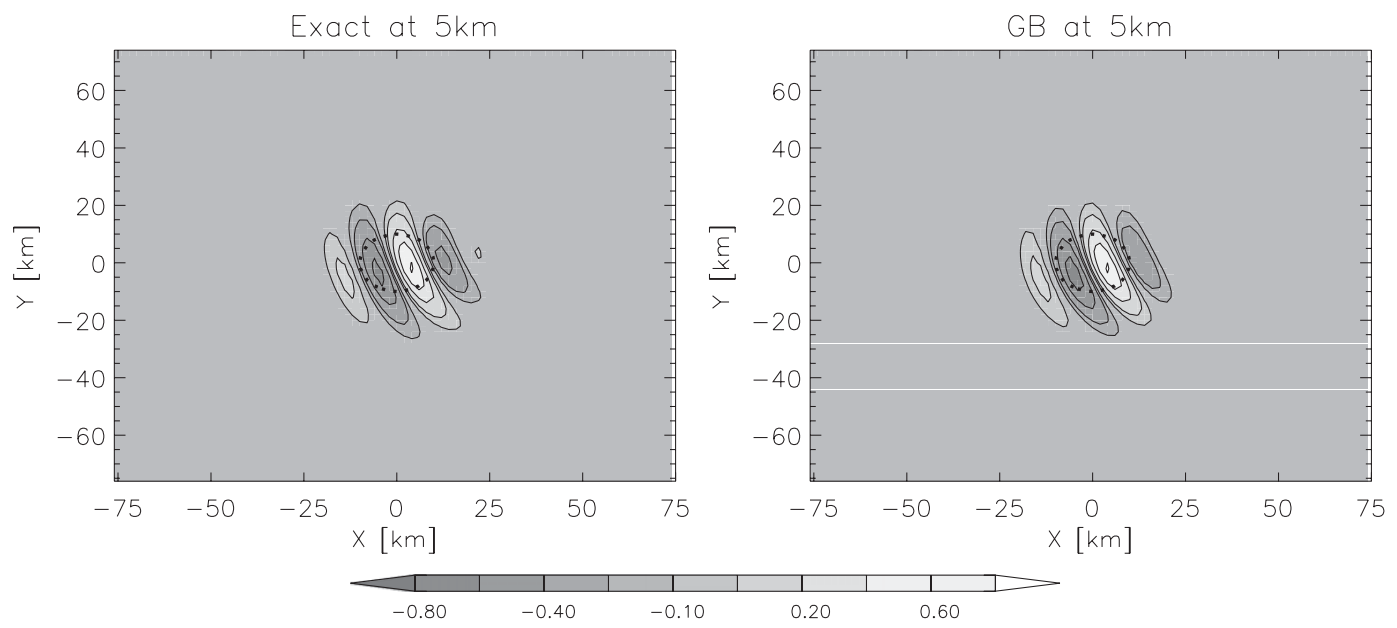

FIG. 4. Horizontal cross section at a height of $5 \mathrm{~km}$ of the vertical displacement $\left(\eta / \eta_{c}\right)$ of the wave disturbance in the presence of a caustic at $5 \mathrm{~km}$ : (left) the exact solution and (right) the Gaussian beam approximation. 

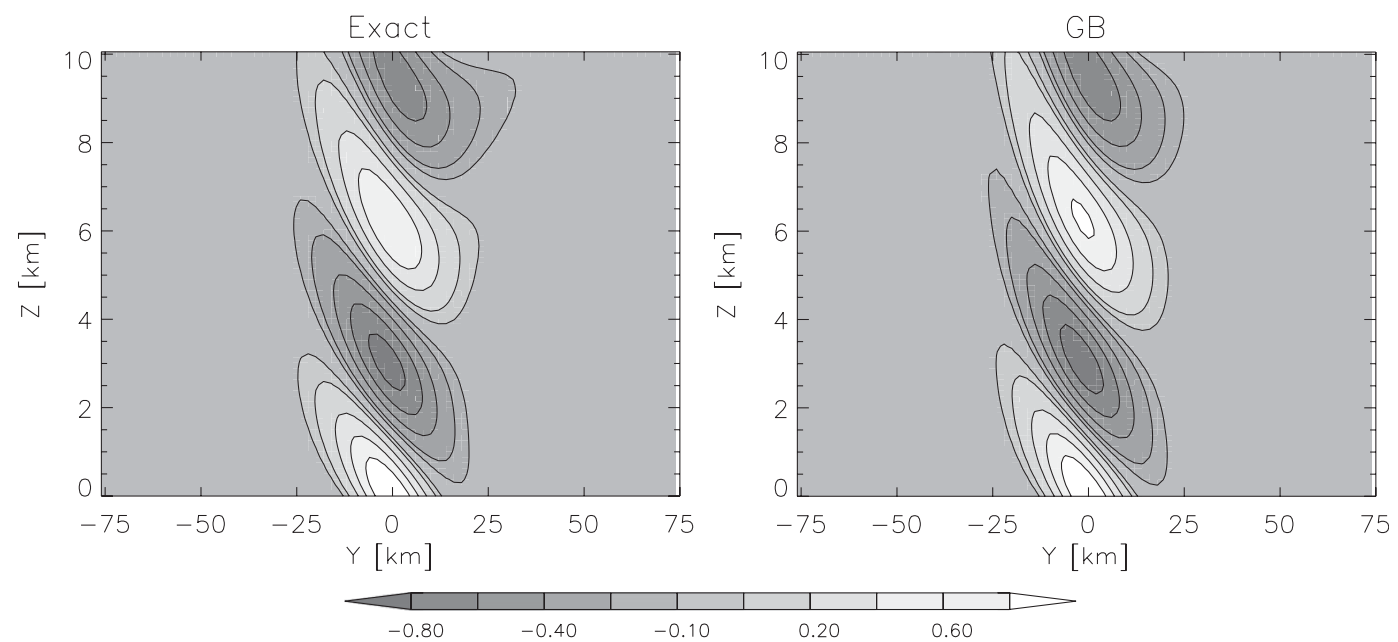

FIG. 5. Vertical-meridional cross section of the vertical displacement $\left(\eta / \eta_{c}\right)$ at $x=0 \mathrm{~km}$ in the presence of a caustic at $5 \mathrm{~km}$ : (left) the exact solution and (right) the Gaussian beam approximation.

calculate the spectrum. Then, the amplitude of the Gaussian is determined from

$\hat{\eta}_{c i j}=\left(\pi \sigma_{k} \sigma_{l}\right)^{-1 / 2}\left[\int_{k_{c i}-\Delta k / 2}^{k_{c i}+\Delta k / 2} \int_{l_{c i}-\Delta l / 2}^{l_{c i}+\Delta l / 2}|\hat{h}(k, l)|^{2} d k d l\right]^{1 / 2}$.

In this way the algorithm conserves wave energy. In general, we establish a threshold of minimum amplitude for the Gaussians: if the amplitude of a particular Gaussian is smaller than the threshold, the Gaussian is not considered to construct the superposition of Gaussian beams.

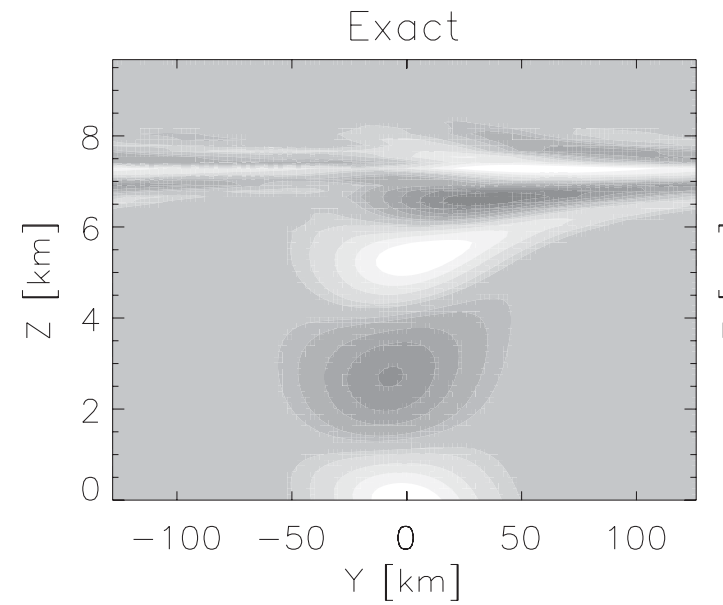

The constant $C$ that determines the width of the Gaussian beams is the only free parameter of the algorithm. We tune it so that the superposition of Gaussians of the form (23) fits optimally the Fourier transform of the orography $\hat{h}(k, l)$. Characteristic values of $C$ are $0.38-0.45$.

\section{1) A BELl IN A CONSTANT WIND}

A rather standard case of a localized mountain is the bell-shaped function (e.g., Smith 1980):

$$
h(x, y)=h_{0}\left[1+a^{-2}\left(x^{2}+y^{2}\right)\right]^{-3 / 2} .
$$

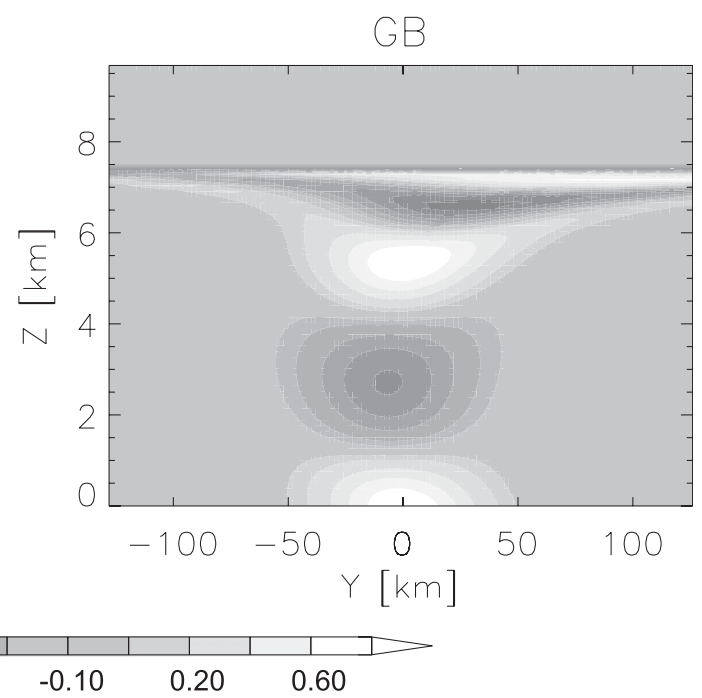

FIG. 6. Vertical cross section at $x=0 \mathrm{~km}$ of the vertical displacement $\left(\eta / \eta_{c}\right)$ generated by a Gaussian beam in a background wind with constant magnitude that turns with height: (left) the exact solution and (right) the Gaussian beam approximation. 


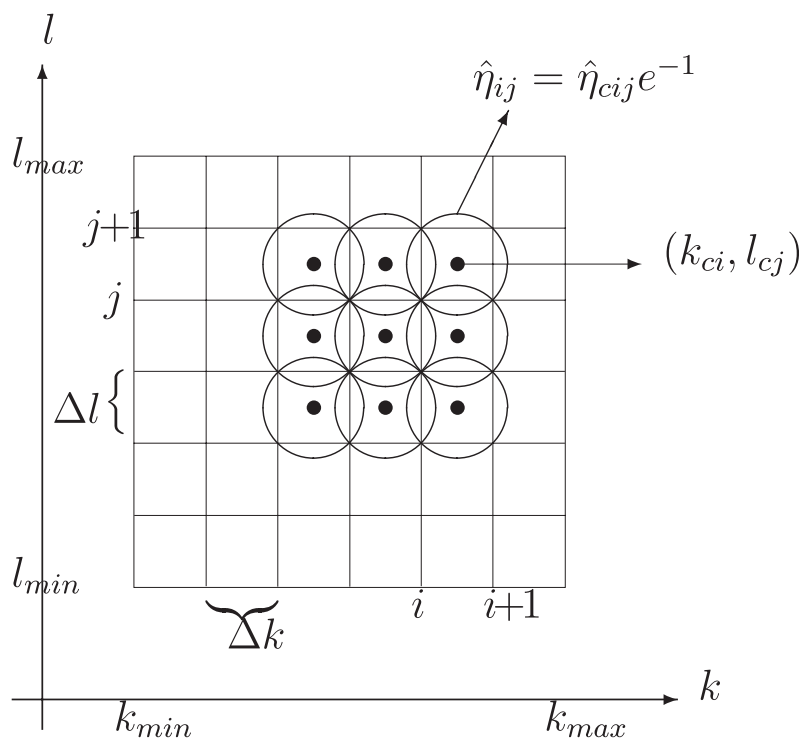

FIG. 7. Wavenumber space grid and the Gaussian locations. See text for details.

The energy of the disturbance at a fixed height is concentrated along the characteristic V-shaped downstream ship-wave pattern. In particular, the pattern of the disturbance forms a parabola with its vertex at the origin (Smith 1980). This parabola is produced by rays of different wavenumbers that compose the disturbance. The longer the wavelengths and the larger the angle to the background wind, the further the wave propagates downstream. At a given height, long-wavelength waves produce the highest amplitudes.

In the hydrostatic approximation this case cannot be modeled with a single Gaussian beam since the second derivative of the phase, $\partial_{k_{i} k_{j}} \phi(i, j=1,2)$, in one of the principal axes vanishes. For the case of a Gaussian beam superposition, we avoid the zero wavenumber as central wavenumber to form the spectrum of the bell-shaped mountain. The spectrum to build the Gaussian beam superposition is obtained by analytically Fourier transforming (28). A cell grid of $20 \times 20$ is used, which covers from $k_{\min }=l_{\min }=-3 a^{-1}$ to $k_{\max }=l_{\max }=3 a^{-1}$.

The (exact) wave field at $z=3.6 U / N$ is shown in the left panel of Fig. 8. The approximated wave field produced by the Gaussian beam superposition can be seen in the middle panel of Fig. 8. The approximation captures quite well the $\mathrm{V}$ shape of the pattern, which is formed by each component of the spectrum. The amplitude is also in general quite well represented. Small differences can be found far from the source (Fig. 8). Two effects may be contributing to these slight differences. The waves that propagate farthest belong to the high-wavenumber part of the spectrum, which is not well represented. The differences could also be produced by high-order dispersive effects that are not represented in the Gaussian beam approximation. Experiments with a larger number of Gaussian beams diminish the differences but do not eliminate them.

The right panel of Fig. 8 shows the stationary phase solution except around the origin where the approximation is not bounded. Along the central ray and far from the origin the stationary phase solution agrees with the exact case. Near the vertical axis the stationary phase solution is not precise; both the amplitude and the phase are not well represented. The highest amplitude of the "exact" disturbance is found near the vertical axis so that this is an essential region to be modeled for a gravity wave drag scheme. On the other hand, the Gaussian beam approximation (middle panel of Fig. 8) is in remarkably good agreement with the exact case (right panel).
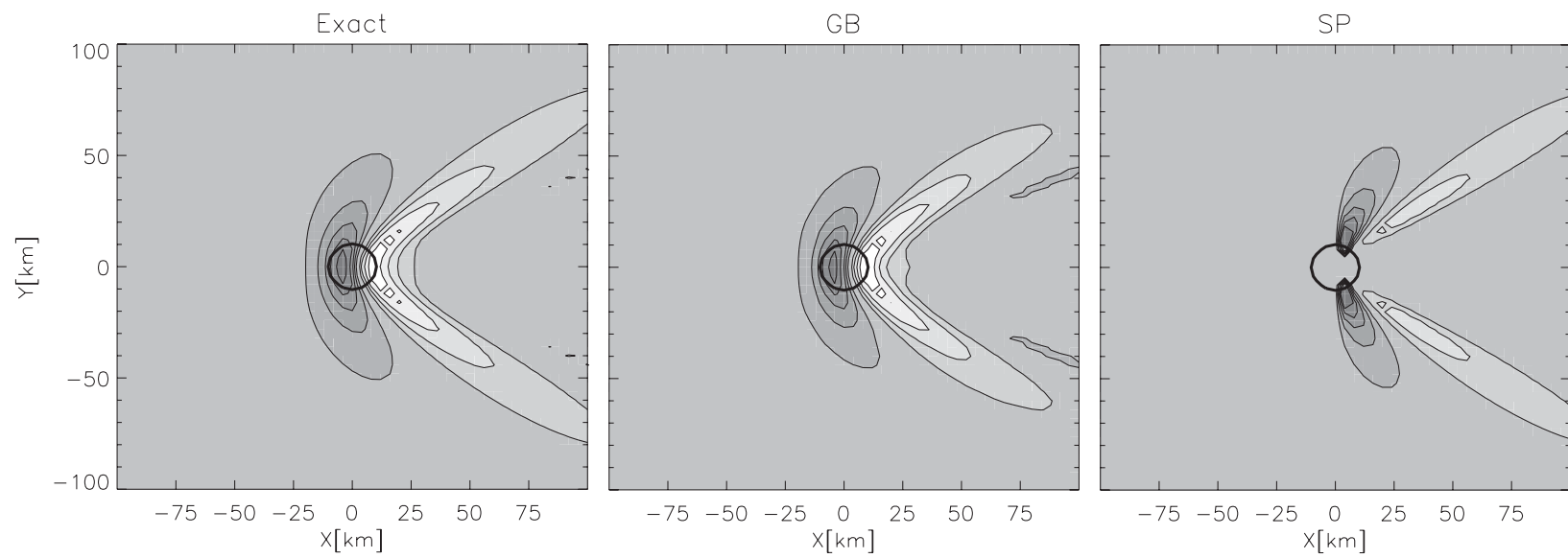

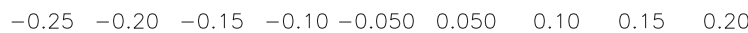

FIG. 8. Vertical displacements $\left(\eta / \eta_{c}\right)$ of the wave disturbance generated by a bell-shaped mountain at $z=3.6 \mathrm{~km}(3.6 U / N)$. 

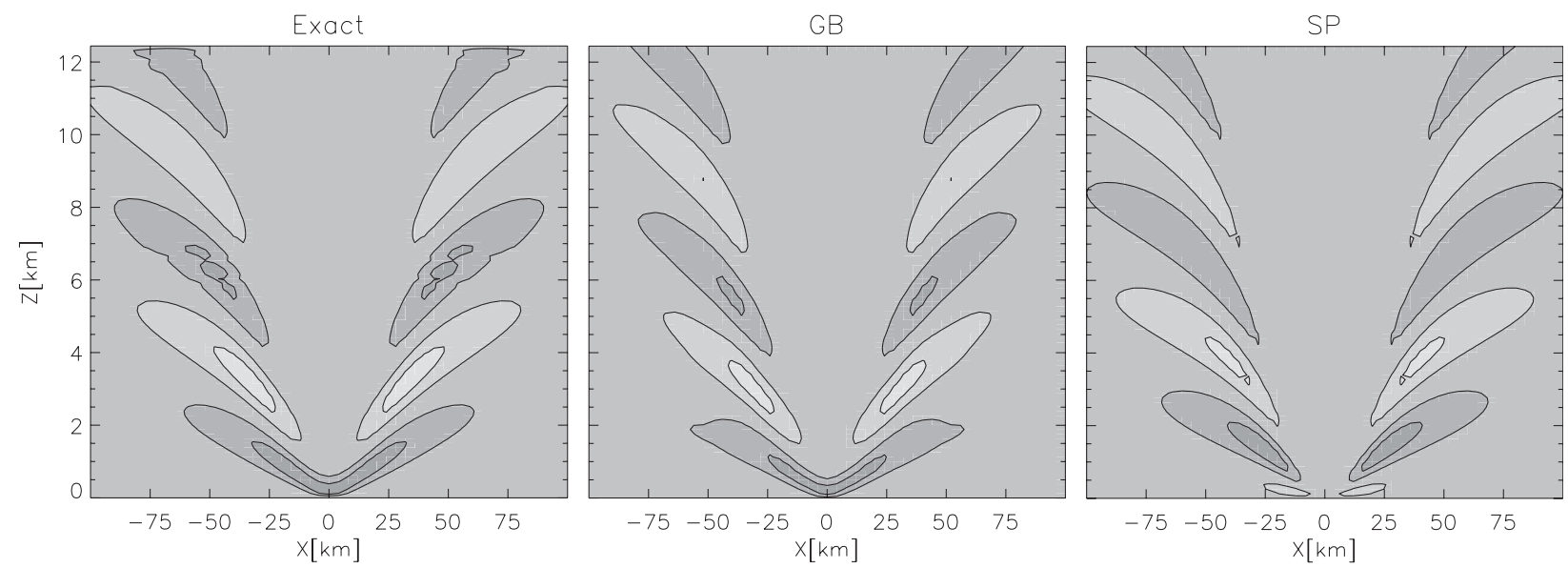

$\begin{array}{lllllllll}-0.25 & -0.20 & -0.15 & -0.10 & -0.050 & 0.050 & 0.10 & 0.15 & 0.20\end{array}$

FIG. 9. As in Fig. 8, but at $x=52 \mathrm{~km}$.

In a $y-z$ cross section upstream from the source, the two branches of the parabola are found, as seen in Fig. 9. The amplitude of the Gaussian beam approximation (middle panel) is slightly lower than the exact solution (left panel). In general, both Gaussian beam and stationary phase approximations (right panel) are in good agreement with the exact solution in this $y-z$ cross section far from the origin $(x=52 \mathrm{~km})$. Some Fourier effects due to numerical wraparound of the solution as a result of periodic boundary conditions are present in the exact solution.

\section{2) Multiple CRITICAL LEVELS REVISED}

In this experiment we model again the multiple critical levels case [see section $3 b(3)$ ], that is, a disturbance generated by orographic forcing representing a Gaussian modulated sinusoidal wave that propagates in a wind turning with height. The disturbance is represented by a superposition of Gaussian beams instead of a single Gaussian beam. A $20 \times 20$ cell grid is used from $k_{\min }=$ $l_{\min }=-3 \sigma_{k}$ to $k_{\max }=l_{\max }=3 \sigma_{k}$.

The perturbation found in the exact case (left panel of Fig. 6) above the critical level of the central ray can be well reproduced by a superposition of Gaussian beams (Fig. 10). There are some slight differences between the exact case and the Gaussian beam approximation because of the difficulty in representing the small vertical wavelengths present in that height range (this is for both cases the exact and the approximation).

The single Gaussian beam case contains only one critical level related to the central wavenumber, so the disturbance is concentrated around the central ray (see left panel of Fig. 6). On the other hand, for a superposition of Gaussian beams each Gaussian beam has its own critical level. The contributions of the Gaussian beams near their critical levels are nonnegligible; thus, the height range of the disturbance envelope for large $s$ is wider than the single Gaussian beam case. This broad height range of the disturbance envelope is found in the exact case (left panel of Fig. 6).

\section{3) A CASE STUDY OF REALISTIC OROGRAPHY}

So far we have examined disturbances generated by simple analytical localized mountains. We now examine orographic waves generated by realistic orography. We take the orography from the tip of South America in the latitudinal range from $45.9^{\circ}$ to $51.9^{\circ} \mathrm{S}$ and in the longitudinal range from $64^{\circ}$ to $80^{\circ} \mathrm{W}$. The orography belongs to the Andes mountain range and presents a plateau with several mountains, two of them reaching altitudes

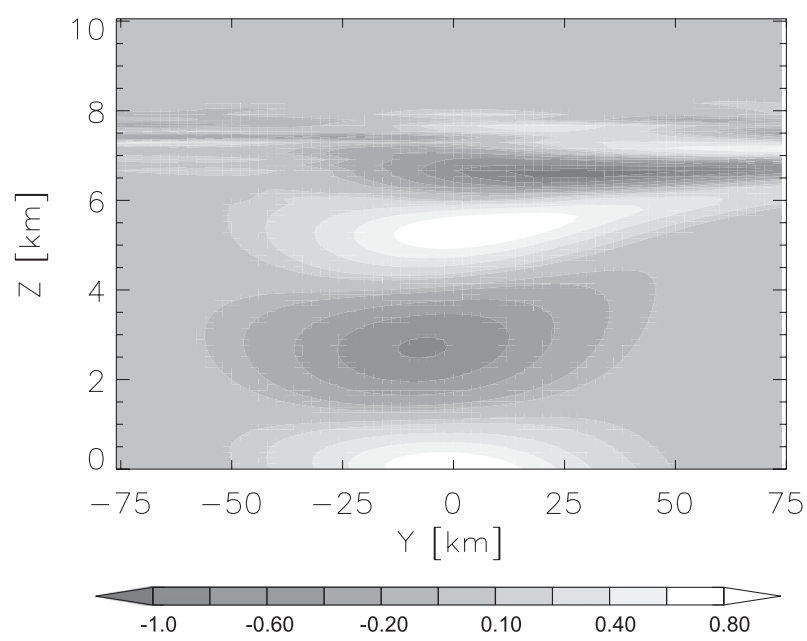

FIG. 10. As in Fig. 6, but for the wave disturbance represented by a superposition of Gaussian beams. See exact solution in the left panel of Fig. 6. 


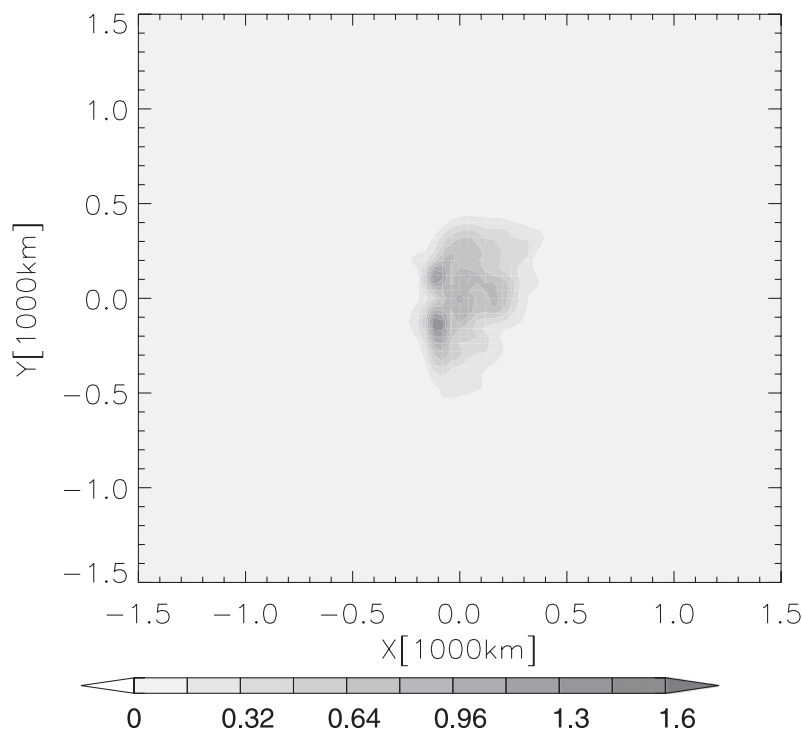

FIG. 11. Orography $(\mathrm{km})$ from the tip of South America in the latitudinal range $45.9^{\circ}-51.9^{\circ} \mathrm{S}$ (after windowing).

higher than $1500 \mathrm{~m}$, see Fig. 11. In low and high latitudes a window has been imposed so that the orography goes smoothly to zero at the boundaries to avoid leakage effects when the orography is Fourier transformed to obtain $\hat{h}(k, l)$.

We again set characteristic background conditions; the background wind is $10 \mathrm{~m} \mathrm{~s}^{-1}$ and the Brunt Väisälä frequency is $10^{-2} \mathrm{~s}^{-1}$. Note that we assume the background wind is uniform. This representation of the wind is only used for evaluation of the technique, but results might differ if horizontal or vertical shear of the background wind were taken into account.
The procedure is equivalent to the one performed for idealized cases, except that the spectrum of the orography is computed using a fast Fourier transform algorithm (in the idealized cases the spectrum is calculated analytically). The limits of the wavenumber space are $k_{\max }=-k_{\min }=$ $l_{\max }=-l_{\min }=8 \times 10^{-5} \mathrm{~m}^{-1}$. Once the Gaussian parameters are known, a superposition of Gaussian beam solutions (21) is computed in the physical domain.

A horizontal cross section at $3.6 U / N$ of the orographic waves generated by the mountain is shown in Fig. 12 . The disturbance is a superposition of the forcing by the large-scale plateau, which generates a deformed parabola similar to analytical cases (e.g., Fig. 8) and small-scale perturbations generated by the peaks. In Fig. 12 the middle panel presents the Gaussian beam superposition with a resolution of $50 \times 50$ grid cells. There are a total of 356 Gaussian beams (spectral amplitudes below a threshold, 0.01 times the maximum amplitude, are not represented). A good representation of the large-scale perturbation is obtained in the pattern and amplitude of the disturbance. There is also good convergence to the exact wave disturbance when increasing the number of Gaussian beams. The right panel shows the disturbance generated by 1447 Gaussian beam solutions with a resolution of $100 \times 100$ grid cells and same threshold as the earlier case. The small-scale features are better represented in this last case. Figure 13 presents a vertical cross section of the disturbance; good agreement between the exact case and the Gaussian beam approximation is also found in the $x-z$ cross section.

\section{Conclusions}

Contrary to the ray tube approximation or the stationary phase method, which are only useful for the far-field

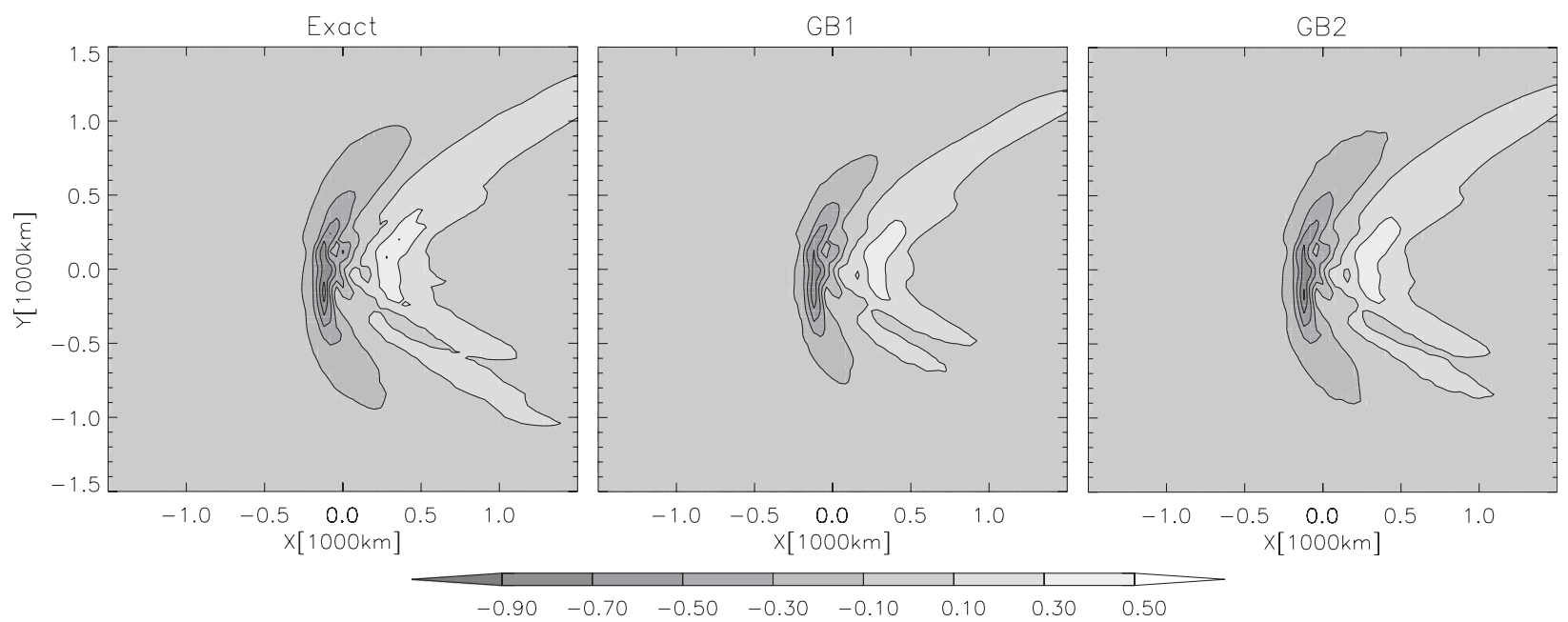

FIG. 12. Vertical displacements $\left(\eta / \eta_{c}\right)$ of the wave disturbance at $3.6 \mathrm{~km}(3.6 U / N)$ : (left) the exact solution and for (middle) 357 and (right) 1447 Gaussian beams. 


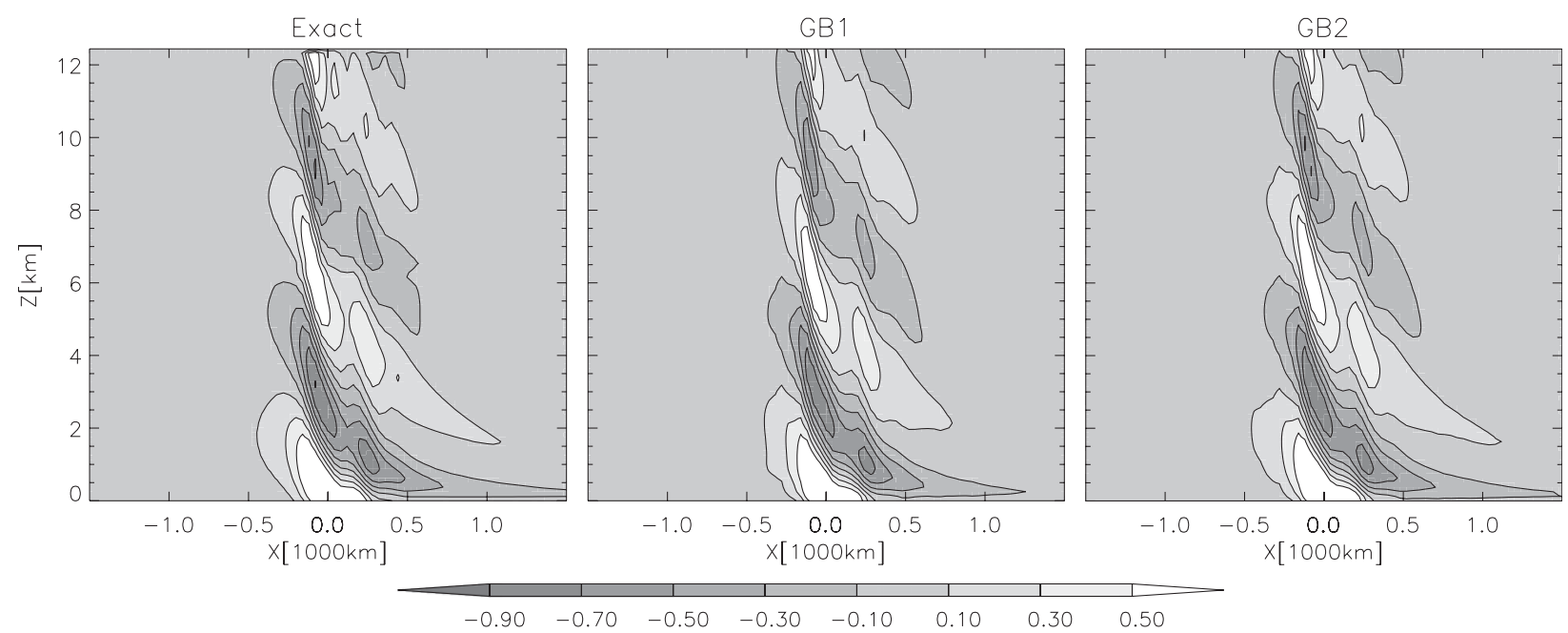

FIG. 13. Vertical displacements $\left(\eta / \eta_{c}\right)$ of the wave disturbance in an $x-z$ cross section at $48^{\circ} \mathrm{S}$ : (left) the exact solution and for (middle) 357 and (right) 1447 Gaussian beams.

representation, the Gaussian beam approximation allows one to represent the wave field both near the source and in the far field. The comparison with the exact linear solution using a numerical model shows that even close to the source (small $s$ ), where a caustic exists, the approximation is precise. This agreement between the exact field and the single-Gaussian beam approximation may be expected as long as the slowly varying wave train assumption is valid for the wave forcing field (the orography).

In this work we propose to represent realistic orography using a superposition of Gaussian beams. The Gaussian beams are set so that the power spectrum of the orography is fitted. As a first approach to set the two parameters, the width and amplitude of the Gaussian, we set the amplitude of the Gaussian so that the algorithm conserves wave energy for each spectral bin and the width is tuned subjectively to represent the power spectrum of the orography as closely as possible. To implement the technique in a wider range of applications, instead of the few case studies presented here, a least squares problem could be designed wherein a cost function that measures the differences between the power spectrum of the orography and the Gaussian beam superposition is defined such that the minimum of the cost function gives the optimum Gaussian width.

Since the main goal of this work is to apply the Gaussian beam approximation to orographic gravity waves and to compare its potential with standard ray theory, the simplest gravity wave dispersion relation is chosen-in particular, hydrostatic and nonrotating approximations are taken-so that agreement and differences are more clearly established. A generalization of the Gaussian beam approximation to include rotation and nonhydrostatic effects is under way and will be published in a subsequent work. The only exceptions that can be envisaged in more realistic dispersion relationships are cases where the second derivatives of the phase are zero so that higher order terms in the Taylor series of the phase [see (19)] must be taken into account.

The Gaussian beam technique is computationally very efficient: it only requires evaluation of functions, that is, (21), and does not require any extra transformation except the initial one to determine $\hat{\eta}(k, l)$. On the other hand, the numerical simulations performed to obtain what we call the "exact solution" are extremely demanding, particularly in the presence of critical levels. In the neighborhood of multiple critical levels there is strong negative interference between modes; indeed, while the individual modes have a singularity at their critical levels, the full wave field amplitude goes to zero there (Shutts 1998). The correct numerical representation of this strong destructive interference requires a very high resolution in wavenumber space (e.g., Pulido 2005; Pulido and Rodas 2008). The Gaussian beam represents well this destructive interference within a ray tube so that only the evaluation of a few Gaussian packets is needed to have a realistic wave amplitude close to multiple critical levels.

If standard ray techniques are used as a gravity wave parameterization, the appearance of caustics could contaminate the gravity wave drag estimation. Since the wave amplitude grows indefinitely near caustics, the wave field will be artificially saturated near caustics. Thus, the parameterization will artificially deposit wave momentum flux in the mean flow. To avoid these inconveniences, current ray techniques (e.g., Marks and Eckermann 1995; 
Hasha et al. 2008) assume that the cross-sectional area of ray tubes is constant along the entire ray. The Gaussian beam approximation gives a wave field that is free of caustics and, therefore, this approximation is particularly suitable for future gravity wave parameterizations.

Acknowledgments. This work was partially funded by a grant from the Agencia Nacional de Promoción Científica y Tecnológica. We thank Peter Hitchcock and two anonymous reviewers for careful reviews and comments that helped to improve the manuscript.

\section{REFERENCES}

Alexander, M. J., and K. H. Rosenlof, 1996: Nonstationary gravity wave forcing of the stratospheric zonal mean wind. J. Geophys. Res., 101, 23 465-23 474.

Bretherton, F., 1969: On the mean motion induced by internal gravity waves. J. Fluid Mech., 36, 785-803.

—_, and C. Garrett, 1968: Wavetrains in inhomogeneous moving media. Proc. Roy. Soc. London, 302A, 529-554.

Broad, A., 1999: Do orographic gravity waves break in flows with uniform wind direction turning with height? Quart. J. Roy. Meteor. Soc., 125, 1695-1714.

Broutman, D., J. Rottman, and S. Eckermann, 2001: A hybrid method for wave propagation from localized source, with application to mountain waves. Quart. J. Roy. Meteor. Soc., 127, 129-146.

— $—$ - and —_, 2002: Maslov's method for stationary hydrostatic mountain waves. Quart. J. Roy. Meteor. Soc., 128, 1159-1171.

— , J. Ma, S. Eckermann, and J. Lindeman, 2006: Fourier-ray modeling of transient trapped lee waves. Mon. Wea. Rev., 134, 2849-2856.

Bühler, O., M. McIntyre, and J. Scinocca, 1999: On shear generated gravity waves that reach the mesosphere. Part I: Wave generation. J. Atmos. Sci., 56, 3749-3763.

Cerveny, V., M. M. Popov, and I. Psencik, 1982: Computation of wave fields in inhomogeneous media-Gaussian beam approach. Geophys. J. Roy. Astron. Soc., 70, 109-128.

Greiner, W., and J. Reinhardt, 1996: Field Quantization. Springer, $440 \mathrm{pp}$.

Hagedorn, G., 1984: A particle limit for the wave equation with a variable wave speed. Commun. Pure Appl. Math., 37, 91-100.

, 1985: Semiclassical quantum mechanics. IV: Large order asymptotics and more general states in more than one dimension. Ann. l'Inst. H. Poincaré, 42, 363-374.

Hasha, A., O. Bühler, and J. Scinocca, 2008: Gravity-wave refraction by three-dimensionally varying winds and the global transport of angular momentum. J. Atmos. Sci., 65, 2892-2906.

Heyman, E., and L. Felsen, 2001: Gaussian beam and pulsed beam dynamics: Complex source and spectrum formulations within and beyond paraxial asymptotics. J. Opt. Soc. Amer., 18A, $1588-1611$.
Hines, C. O., 1997: Doppler spread parameterization of gravitywave momentum deposition in the middle atmosphere. Part 1: Basic formulation. J. Atmos. Sol.-Terr. Phys., 59, 371-386.

Lewis, R. M., 1965: Asymptotic theory of wave-propagation. Arch. Ration. Mech. Anal., 20, 191-250.

Li, F., J. Austin, and J. Wilson, 2008: The strength of the BrewerDobson circulation in a changing climate: Coupled chemistryclimate model simulations. J. Climate, 21, 40-57.

Lighthill, J., 1978: Waves in Fluids. Cambridge University Press, $504 \mathrm{pp}$.

Lindzen, R. S., 1981: Turbulence and stress owing to gravity wave and tidal breakdown. J. Geophys. Res., 86, 9707-9714.

Lott, F., and M. Miller, 1997: A new subgrid-scale orographic drag parametrization: Its formulation and testing. Quart. J. Roy. Meteor. Soc., 123, 101-127.

Marks, C., and S. Eckermann, 1995: A three-dimensional nonhydrostatic ray-tracing model for gravity waves: Formulation and preliminary results for the middle atmosphere. J. Atmos. Sci., 52, 1959-1984.

Martin, A., and F. Lott, 2007: Synoptic responses to mountain gravity waves encountering directional critical levels. $J$. Atmos. Sci., 64, 828-848.

McFarlane, N., 1987: The effect of orographically excited gravity wave drag on the general circulation of the lower stratosphere and troposphere. J. Atmos. Sci., 44, 1775-1800.

McLandress, C., and T. Shepherd, 2009: Simulated anthropogenic changes in the Brewer-Dobson circulation, including its extension to high latitudes. J. Climate, 22, 1516-1540.

Ostrovsky, L. A., and A. I. Potapov, 1999: Modulated Waves: Theory and Applications. The John Hopkins University Press, 369 pp.

Palmer, T. N., G. J. Shutts, and R. Swinbank, 1986: Alleviation of a systematic westerly bias in general circulation and numerical weather prediction models through on orographic gravity wave drag parametrization. Quart. J. Roy. Meteor. Soc., 112, 1001-1039.

Popov, M. M., 1982: A new method of computation of wave fields using Gaussian beams. Wave Motion, 4, 85-97.

Pulido, M., 2005: On the Doppler shifting effect in an atmospheric gravity wave spectrum. Quart. J. Roy. Meteor. Soc., 131, 12151232.

- and C. Rodas, 2008: Do transient gravity waves in a shear flow break? Quart. J. Roy. Meteor. Soc., 134, 1083-1094.

- and J. Thuburn, 2008: The seasonal cycle of gravity wave drag in the middle atmosphere. J. Climate, 21, 4664-4679.

Scinocca, J., 2003: An accurate spectral nonorographic gravity wave drag parameterization for general circulation models. J. Atmos. Sci., 60, 667-682.

Shutts, G. J., 1998: Stationary gravity-wave structure in flows with directional wind shear. Quart. J. Roy. Meteor. Soc., 124, 14211442 .

Smith, R. B., 1980: Linear theory of stratified hydrostatic flow past an isolated mountain. Tellus, 32, 348-364.

Tanushev, N. M., J. Qian, and J. V. Ralston, 2007: Mountain waves and Gaussian beams. Multiscale Model. Simul., 6, 688-709.

Webster, S., A. R. Brown, D. R. Cameron, and C. P. Jones, 2003: Improvements to the representation of orography in the Met Office Unified Model. Quart. J. Roy. Meteor. Soc., 129, 1989-2010. 